\title{
Augmented Reality (AR) - based Human Digestive System Mobile Learning Platform
}

\author{
Akinyokun Oluyomi \\ Department of Computer Science \\ The Federal University of Technology, Akure \\ Ondo State, Nigeria
}

\author{
Aladeselu Oluwamodupe \\ Department of Computer Science \\ The Federal University of Technology, Akure \\ Ondo State, Nigeria
}

\begin{abstract}
Overtime, the inadequacy and abstraction of conventional teaching materials had been a bane of bringing the interest of students to learning. Likewise, teaching method driven by abstraction may often not seem appealing to the targeted audience. Thus computer-aided teaching such as the use of augmented reality serves as an alternative to effective and efficient teaching. This research presents a $3 \mathrm{D}$ anatomical representation of the human digestive system using Augmented Reality (AR) thus creating a paradigm for students to have a better picture and understanding of the human anatomy and how human body parts are connected. Drawbacks such as inaccessibility to cadaver due to ethics/regulations and anxiety/nausea when participating in such sessions can be reduced or controlled using AR through computer-aided graphics to add an additional layer of information for better interaction and understanding. The system also presents a real-time direct or indirect view of a real world environment that has been augmented. Students also get the chance to play quiz game to examine their knowledge on digestive system.
\end{abstract}

\section{General Terms}

Augmented Reality Mobile learning platform

\section{Keywords}

Augmented Reality, Human Anatomy, Digestive system

\section{INTRODUCTION}

Technology has become one of the most supporting tools in education and the results indicate a strong positive influence on learning and teaching styles. According to [12], lessons that are supported by technology will lead to more innovation forms of teaching and learning. The incorporation of technology tools into the curriculum is becoming part of good teaching. The integration of technology provides a means to enhance student learning and engagement in lectures. Augmented Reality (AR) is one of the most recent technological advances that could be used as creativity in promoting educational tool that enables users to see and experience the real world mixed with diverse virtual objects, without losing the sense of reality.

Augmented Reality has been around for over 50 years and it still seems as a new technology. AR first came onto the scene in the 1960's developed by Ivan Sutherland at the University of Utah and Harvard University. Since this first creation, AR has been further developed in a variety of fields with numerous applications. AR is a complex system that, in a simple explanation, involves a target activating the virtual trigger which will introduce virtual presences into users' reality. This target can be a geo-location, voice activated, a patterned marker, or object recognition. The most common methods for displaying the virtual aspect of Augmented Reality are through projection using head-mounted displays, see-through display, and through handheld devices. With handheld devices, AR apps are deployed to smart phones and tablets and typically interact with targets that are printable markers or images, similar to a QR code [4].

Students commonly find Science subjects to be abstract, requiring a depth of understanding and visualization skills [3]. When students have difficulties in understanding the concept well, it leads to misconceptions. According to [11], misconception among students has to be taken into consideration because it can interfere with the students' learning of scientific principles and concepts. Thus, the selection of teaching method plays an important factor in minimizing or avoiding the students' misconception [11]. Visualization technologies have exciting potential for preventing misconceptions in the scientific domain. There is a wide range of technologies made available that can be used for the visualization of abstract concepts. Nowadays, one of the technologies that have great potential in education especially in visualizing abstract concepts is Augmented Reality.

Human anatomy is the study of the human body's structure and its parts. Learning human anatomy is one of the most vital and necessary parts of any aspiring medical student. It gives students a good grasp on how the different parts of the human body are connected and often serves as the foundation for more specialized studies. Anatomical learning have traditionally been taught by dissecting human or animal bodies in order to give students a hands on experience and better understanding of the human anatomy. While using real cadavers is usually a very informative and valuable experience for any aspiring medical student, there are multiple drawbacks with this method.

Students cannot easily access and study a cadaver whenever they want. Access to such material is usually strictly controlled and regulated. Students have also reported experiencing anxiety or nausea when participating in such sessions.

This research will address the issue of misconception among students in studying the digestive systems and also, help medical students in better understanding of the Human digestive system without having to dissect human bodies.

\section{LITERATURE REVIEW}

Augmented reality, also called AR has gained significant attention in recent years. This terminology called AR has been used to describe the technology behind the enlargement or enhancement of the real world. To "augment reality" is to 
"intensify" or "expand" reality itself. Specifically, AR is the ability to superimpose virtual objects on to the real world through the screen of a device such as a personal computer or a smart phone, to create and show users a world full of information [10].

Augmented Reality have the following characteristics:

1. It combines real and virtual environments.

2. It is interactive.

3. It is registered in three - dimension.

\subsection{Reality-Virtuality Continuum}

Milgram et al. [8], introduced the reality-virtuality continuum that defines mixed reality and it is said to be the link between the real and the virtual world. If the real world is at one of the ends of the continuum and Virtual Reality is at the other end, then the Augmented Reality takes up the space closer to the real world. The closer a system is to the Virtual Environment, the more the reality of the object decreases. If the real world can be augmented with virtual objects, it is coherent to expect that the virtual world can be augmented with real views, and objects. Such environment is called Augmented Virtuality
(AV). On the reality-virtuality continuum, Augmented Virtuality takes up the space closer to the Virtual Reality environments.

Augmented virtuality (AV), is a subcategory of mixed reality which refers to the merging of real world objects into virtual worlds. It is simply a virtual world with a bit of reality in it.

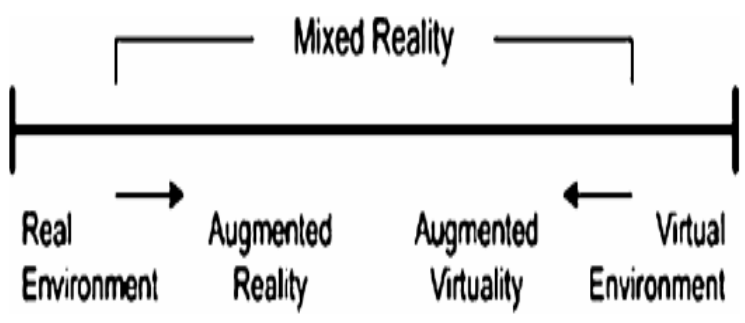

Fig 1: Milgram's Reality-Virtuality [8]

An AR system basically consists of a camera, a processor and a display unit. The camera captures an image, and then AR software system superimposes virtual objects onto the image in real time and displays the result (Siltanen, 2012).

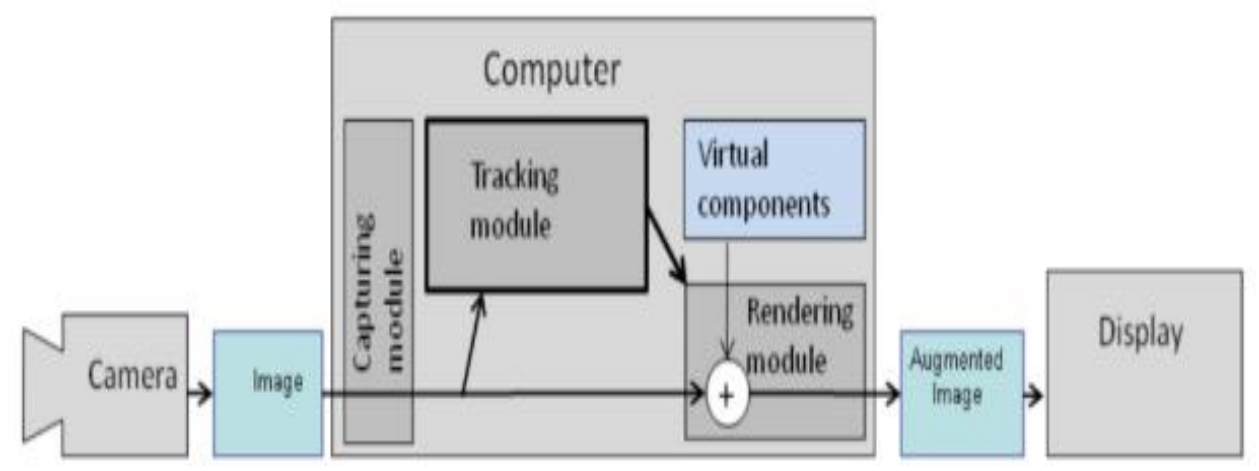

Fig 2: A simple AR system [13]

As shown in the above figure, the current image viewed by the camera is captured by the image acquisition module. The tracker calculates the correct orientation and location for virtual overlay relative to the camera. The renderer finally puts the virtual image on top of the real image using the pose that has been calculated and renders the augmented image onto the display. The tracker is the most critical component here, since its role is to compute the relative pose of the camera in real time. The term "pose" refers to the six degrees of freedom (DOF) orientation and position. The fundamental difference compared to other image processing tools is that in $\mathrm{AR}$ virtual objects are moved and rotated in $3 \mathrm{D}$ coordinates instead of 2D image coordinates.

\section{MATERIALS AND METHODS}

This study is aimed at designing and implementing augmented reality model of the human digestive system. It helps students to study the 3D anatomical structure of the human digestive system. For the system to be an effective and interactive, the students were provided with correct information about each component of the digestive system. Students also get the chance to play quiz game to examine their understanding of the digestive system. Atlas and textbooks of anatomy was studied to understand the structure of the digestive system which helped in proper modelling of the digestive system, and also in understanding how the digestive system works. There is a pop-label on each of the component that describes the function of each of the components.

\section{SYSTEM DESIGN}

Vuforia Target Management System (VTMS) is a web-based tool that enables developers to create and manage image targets (markers) and is saved in the application asset. It acts as a recognition system that compares requests from the mobile application with the image targets in the device storage to find a match, and once a match is found, the appropriate information associated to the target is returned as response. Augmented reality markers that has been scanned are sent to the VTMS as requests corresponding data is returned as response to the requests. 


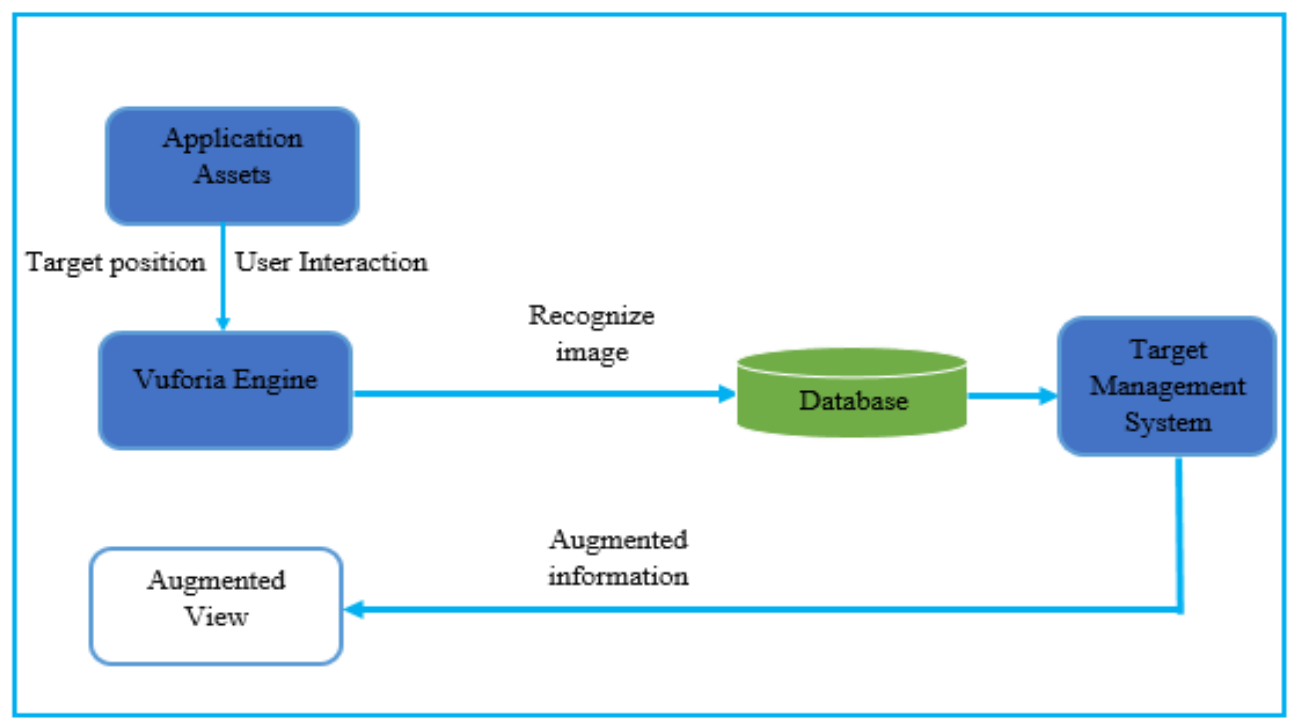

Fig 3: System Architecture

\subsection{System Use Case Diagram}

The use case diagram presents the various activities that can be performed by the user of the system. The description of the use case diagram is given as follows:

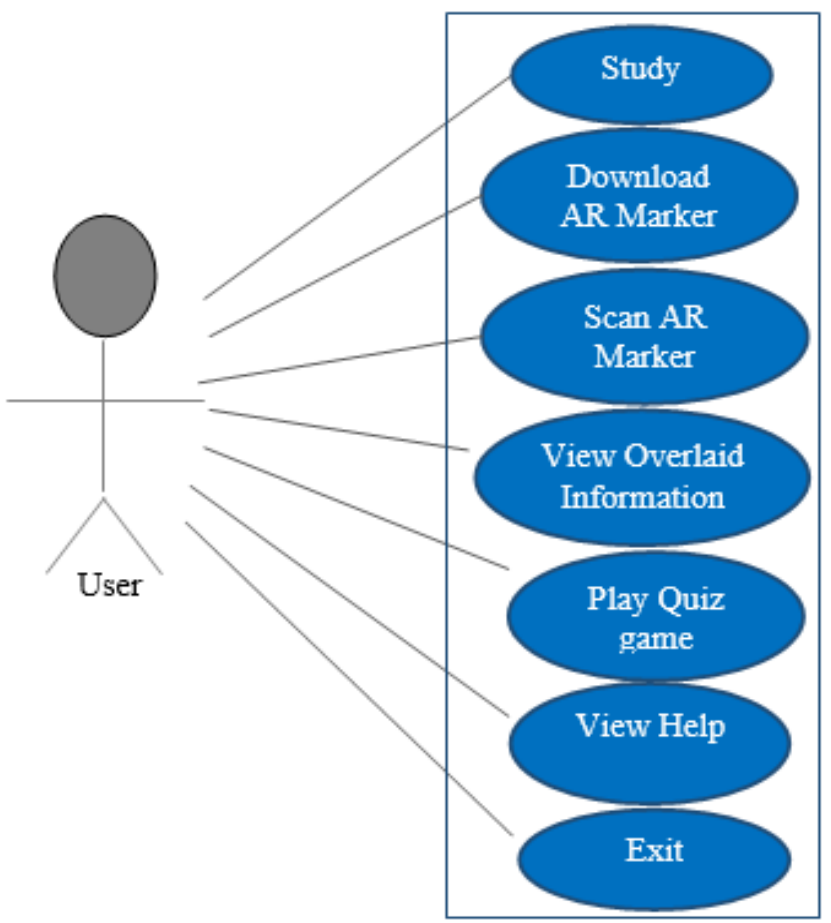

Fig 4: System use case diagram

i. Study: This use case is called when the user want to study the digestive system anatomy, which includes the functions of each component of the digestive system and the anatomy.

ii. Download Marker: This use case is called when the user want to download the target image that will be used for tracking.

iii. Scan AR Marker: This use case is called when the user want to scan the image target after it has been successfully downloaded.

iv. View Overlaid Information: This use case is called when the user want to view the information that has been overlaid. v. Play Quiz game: This use case is called when the user play to play a quiz game on digestive system to test their knowledge of the understanding of what they have learnt.

vi. View Help: This use case is called when the user want to view help information.

vii. Quit: This is a use case that terminates the application.

The mathematical representation for camera transformation in augmented reality has been presented in [13] as follows

The transformation $\mathrm{T}$ between a camera and a marker is given as

$$
\mathrm{x}=\mathrm{TX}, \quad(1)
$$

where $\mathrm{X}$ represents a point in world coordinates,

$\mathrm{x}$ represents projection in ideal image coordinates and

$\mathrm{T}$ represents the camera transformation matrix

Transformation $\mathrm{T}$ consists of translation vector $\mathrm{t}$ and $3 \times 3$ rotation matrix $\mathrm{R}$, and can be expressed in matrix form

$$
\mathrm{X}=[\mathrm{R} \mid \mathrm{t}] \mathrm{X}
$$

and can be represented in homogeneous coordinates as

$$
\left[\begin{array}{l}
\mathrm{x} \\
\mathrm{y} \\
\mathrm{z}
\end{array}\right]=\left[\begin{array}{cccc}
\mathrm{r}_{1} & \mathrm{r}_{2} & \mathrm{r}_{3} & \mathrm{t}_{\mathrm{x}} \\
\mathrm{r}_{4} & \mathrm{r}_{5} & \mathrm{r}_{6} & \mathrm{t}_{\mathrm{y}} \\
\mathrm{r}_{7} & \mathrm{r}_{8} & \mathrm{r}_{9} & \mathrm{t}_{\mathrm{z}}
\end{array}\right]\left[\begin{array}{c}
\mathrm{X} \\
\\
\mathrm{Y} \\
\\
\mathrm{z}
\end{array}\right]
$$

\section{SYSTEM IMPLEMENTATION}

The system was implemented using Vuforia SDK which allows creation of the Augmented Reality features, C\# was the programming language used, and android SDK and JDK 
allows the building of the application to android devices. The system consist of five different interfaces.

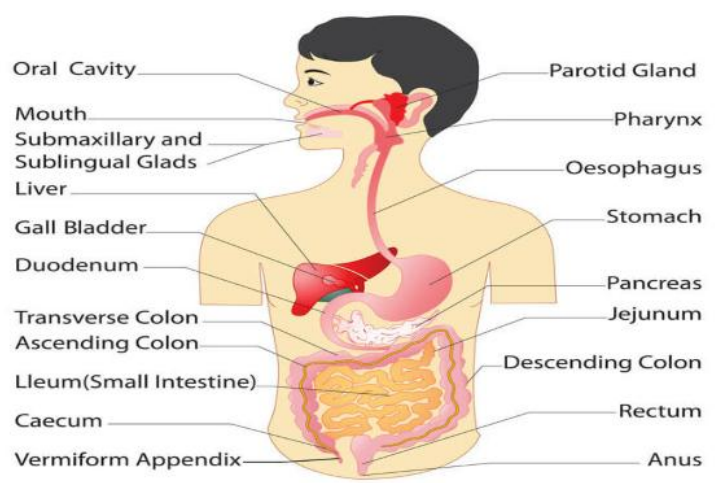

Fig 5: Target image/AR Marker used to augment the digestive

i. The Main-Menu Interface: The Main-Menu interface is the first page the interface see when the application is been launched. The page has different buttons that the user can interact with.

ii. The Study Interface: The study button allows the user to navigate to the study interface. This is the interface that the students can visit to learn about the digestive system. It introduces the students to the digestive system, viewing the digestive system in $3 \mathrm{D}$, explains the basic functions of each component of the digestive system, explains the anatomy of the basic component of the digestive system, students can also view the process of digestion in 3D.

iii. The Quiz Interface: This interface helps to test the knowledge of the students about their understanding of the digestive system after studying. The quiz is a small assessment used in this app to measure growth of knowledge.

iv. The Help Interface: This interface provides all the details the students need to be able to make use of the application efficiently and without stress. The guidelines on how to use the app is provided in this interface. The target image/marker for the application can be downloaded from this interface.

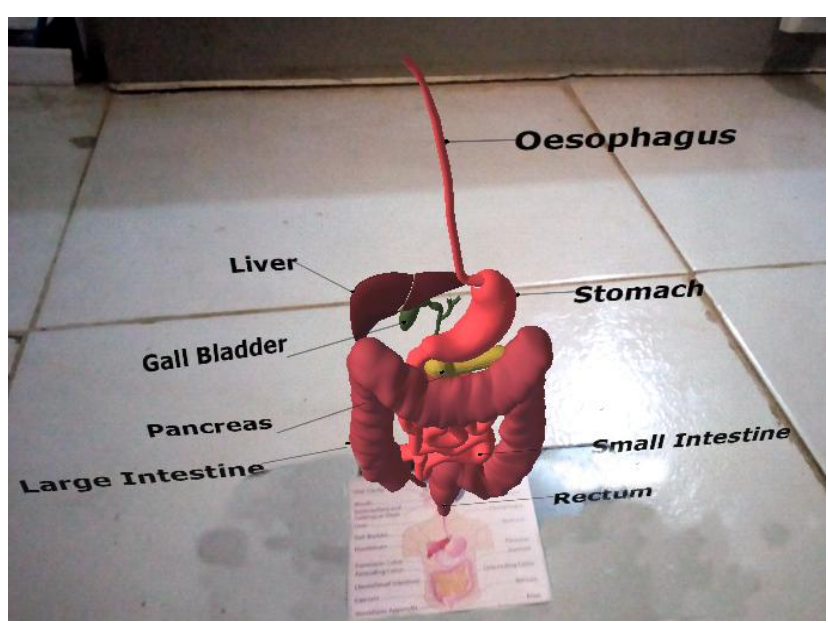

Fig 6: Screenshot of the augmented digestive system

\section{CONCLUSION}

This research is able to implement an augmented reality application that overlays 3D model of the digestive system to the user's environment. Vuforia Target Recognition System (VTRS) was employed and it uses the marker-based technique for tracking. If teachers and lecturers acquire the Augmented Reality Technology for classroom teaching, then the result will be incredible. At the end of this project, an Augmented Reality application was developed and it is expected to help mainly medical students in studying the anatomy of the digestive system.

\section{REFERENCES}

[1] Chien-Huan Chien, C.H.C.S. 2010 An Interactive Augmented Reality System for Learning Anatomy Structure. Proceedings of the International MultiConference of Engineers and computer scientists, Hong Kong.

[2] Egui Zhu, A.H. 2014 Augmented reality in healthcare education: an integrative review. Department of Learning, Informatics, Management and Ethics (LIME), Karolinska Institute, Stockholm Sweden.

[3] Gilbert, J. K. 2004 Models and Modelling: Routes to More Authentic Science Education. International Journal of Science and Mathematics Education.

[4] Herron, J. 2016 Augmented Reality in Medical Education and Training. Journal of Electronic Resources in Medical Libraries

[5] Julie, C., B. 2011 Handbook of Augmented Reality. Department of Computer and Electrical Engineering and Computer Sciences, Florida Atlantic University, Boca Raton, pp. 1-12. Florida.

[6] Margarita, V. 2009 Influence of augmented reality technology upon pupils' knowledge about human digestive system: The results of the experiment. USChina Education Review, Siauliai.

[7] Matina, K. 2015 Augmented Reality for the Study of Human Heart Anatomy. International Journal of Electronics Communication and Computer Engineering.

[8] Milgram, P. T. 1994 Augmented Reality: A Class of Displays on the Reality-Virtuality Continuum. Telemanipulator and Telepresence Technologies. Boston.

[9] Mimaroglu, O. 2014) Collaborative Augmented Reality. Department of Computer Science National University of Ireland, Maynooth Co. Kildare, pp, 1-10. Ireland.

[10] Oluwaranti, A.I. 2015 Architectural Model For An Augmented Reality Based Mobile Learning Application. Journal of Multidisciplinary Engineering Science and Technology, Ile-Ife.

[11] Palmer, D. 2001 Students Alternative Conceptions and Scientifically Acceptable Conceptions about Gravity, International Journal of Science Education.

[12] Shapley, K., S.W. 2011 Effects of technology Immersion on Middle School Students' Learning Opportunities and Achievement. The Journal of Educational Research. 
International Journal of Computer Applications (0975 - 8887)

Volume 174 - No. 32, April 2021

[13] Siltanen, S. 2012 Theory and applications of markerbased augmented reality

[14] Tsai, C. J.-C. 2014 Augmented Reality Application of Multimedia Technology in Aquatic Organisms Instruction. Journal of Software Engineering and Applications, pp. 745-755.
[15] Yeom, S. 2011) Augmented Reality for Learning Anatomy. Proceedings ascilite Hobart 2011, pp. 13771383. Australia.

[16] Zhigang, X., R. A, 2000 Schaum's outlines computer graphics. 